\title{
Preoperative Radiotherapy Leads to Significant Differences in the Plasma Protein Profile of Rectal Cancer Patients
}

\author{
Matilda Holm ${ }^{a-d}$ Sakari Joenväärä ${ }^{e, f}$ Mayank Saraswat ${ }^{g}$ \\ Tiialotta Tohmolae,f,h Ari Ristimäki $^{b, d, f}$ Risto Renkonen ${ }^{e, f} \quad$ Caj Haglund $^{a, c, f}$ \\ aDepartment of Surgery, Faculty of Medicine, University of Helsinki and Helsinki University Hospital, Helsinki, \\ Finland; bepartment of Pathology, Faculty of Medicine, University of Helsinki and Helsinki University Hospital, \\ Helsinki, Finland; 'Translational Cancer Medicine Research Program, Faculty of Medicine, University of Helsinki, \\ Helsinki, Finland; dApplied Tumor Genomics Research Program, Faculty of Medicine, University of Helsinki, Helsinki, \\ Finland; eTransplantation Laboratory, Haartman Institute, University of Helsinki, Helsinki, Finland; ${ }^{f} H U S L A B$, Helsinki \\ University Hospital, Helsinki, Finland; 9Department of Laboratory Medicine and Pathology, Mayo Clinic, \\ Rochester, MN, USA; hDepartment of Biosciences, Faculty of Biological and Environmental Sciences, University of \\ Helsinki, Helsinki, Finland
}

\author{
Keywords \\ Colorectal cancer · Plasma · Proteomics · Preoperative \\ radiotherapy $\cdot$ Mass spectrometry
}

\begin{abstract}
Introduction: Colorectal cancer (CRC) is the third most common cancer worldwide, accounting for $10 \%$ of the global cancer burden. Rectal cancer accounts for around 30\% of CRC cases, and patients with resectable rectal cancer are often given preoperative radiotherapy (PRT) to reduce the rate of local recurrence. The human plasma proteome is an exceptionally complex proteome and ideal to study due to its ability to reflect the presence of diseases such as cancer and the ease of obtaining blood samples. Previous proteomic studies involving rectal cancer patients have mostly focused on the identification of proteins involved in resistance to radiotherapy. Objective: The aim of this study was to investigate the overall effects of PRT on plasma protein expression in rectal cancer patients, as there is a lack of such studies. Methods: Here, we have used mass spectrometry and sub-
\end{abstract}

\begin{tabular}{ll}
\hline karger@karger.com & (c) 2020 The Author(s) \\
www.karger.com/ocl & Published by S. Karger AG, Basel \\
& This article is licensed under the Creative Commons Attribution- \\
Narger & NonCommercial-NoDerivatives 4.0 International License (CC BY- \\
NC-ND) (http://www.karger.com/Services/OpenAccessLicense). \\
Usage and distribution for commercial purposes as well as any dis- \\
tribution of modified material requires written permission.
\end{tabular}

sequent statistical analyses to analyze the plasma samples of 30 rectal cancer patients according to PRT status (positive or negative) and tumor stage (II or III). Results and Conclusions: We discovered 42 proteins whose levels differed significantly between stage II and III rectal cancer patients who did or did not receive PRT. This study shows that PRT, although localized to the pelvis, leads to measurable, tumor stage-specific changes in plasma protein expression. Future studies of plasma proteins should, when relevant, take this into account and be aware of the widespread effects that PRT has on the plasma proteome.

(C) 2020 The Author(s)

Published by S. Karger AG, Basel

\section{Introduction}

In 2018, an estimated more than 1.8 million new cases of colorectal cancer (CRC) were diagnosed, and almost 900,000 deaths occurred. These statistics make CRC the third most common cancer worldwide and the second most common cause of cancer death, second to only lung 
cancer. CRC therefore accounts for around $10 \%$ of the global cancer burden [1]. The term CRC encompasses cancer in both the colon and rectum, although rectal cancer differs from colon cancer in several ways. The rectum is located in the pelvis, a narrower space than the abdomen, which makes surgical resection more challenging for rectal cancer patients and increases the chance of local recurrence. Patients with rectal cancer are therefore often given neoadjuvant (preoperative) radiotherapy or chemoradiotherapy, whereas patients with colon cancer proceed directly to surgery. Rectal cancer accounts for approximately $30 \%$ of all CRC cases $[2,3]$.

Local recurrence is a serious problem for patients with rectal cancer as it causes debilitating symptoms and is hard to treat, and the incidence of local recurrence can be quite high. Preoperative radiotherapy (PRT) has been shown to reduce the rate of local recurrence in patients with resectable rectal cancer. It is usually given in doses of 5 Gy per day over 5 days (short-term PRT), followed by surgery [4]. As found by the Swedish Rectal Cancer Trial, PRT also improves survival for rectal cancer patients, which is explained by the reduced risk of local recurrence. The combination of PRT and surgery has been shown to significantly improve both overall and cancerspecific survival for rectal cancer patients as opposed to surgery alone $[5,6]$.

The proteome is defined as all the proteins expressed in a cell, tissue, or organism. Proteomic techniques are used to analyze various characteristics of proteins, such as their identity, abundance, and function. The proteome is complex, due to the multitude of available posttranslational modifications that proteins can undergo, and dynamic, as protein expression changes during processes such as development and disease [7]. The human plasma proteome is exceptionally complex, as it contains other tissue proteomes as subsets. The ease of obtaining blood samples coupled with the aforementioned complexity and dynamic nature of the proteome makes the plasma proteome ideal to study. It can reflect the presence of diseases such as cancer, which can for example lead to aberrantly secreted proteins that can be detected in the plasma $[8,9]$.

Previous mass spectrometric proteomic studies that have analyzed the proteome of cancer patients receiving radiotherapy have to a large extent focused on attempts to identify proteins that could be used as biomarkers to guide the use of radiotherapy by predicting patient response $[10,11]$. Several studies involving the proteomic profiling of rectal cancer patients have also identified proteins implicated in resistance to radiotherapy, such as acid ceramidase, or protein signatures predictive of radiotherapy response [12-16]. However, there is a paucity of studies with focus on investigating the overall effects of radiotherapy on plasma protein expression in rectal cancer patients.

The aim of this study was to analyze how widespread the effects of PRT are on plasma protein expression in rectal cancer patients. Seeing as CRC is the third most common cancer worldwide, with its prevalence only predicted to increase, further studies are needed in order to better understand different aspects of this heterogeneous disease [17]. The patients were divided into groups based on PRT status (positive or negative), as well as tumor stage (II or III) in order to more accurately analyze plasma protein expression in a stage-dependent manner. In this study, we have analyzed the effects that PRT has on the plasma proteome of rectal cancer patients, and discovered significant differences in plasma protein profiles depending on PRT status.

\section{Material and Methods}

\section{Patient Samples}

This study used plasma samples preoperatively (post-radiotherapy but pre-surgery) obtained from 30 rectal patients (after the exclusion of one sample, marked in online supplementary Table S1 [see www.karger.com/doi/10.1159/000505697], that was not properly digested). The patients in the PRT-positive group received preoperative short-course radiotherapy with a total dose of 25 Gy administered over 5 days $(5 \times 5$ Gy), after which they proceeded immediately to surgery. Plasma samples were collected on the day of the operation from all patients, including those that did not receive PRT. The patients underwent surgical resection at the Department of Surgery, Helsinki University Hospital, between 2000 and 2005. Samples were stored at $-80^{\circ} \mathrm{C}$ until processed. Patients with HNPCC, FAP, ulcerative colitis, Crohn's disease, or mucinous tumors were deliberately excluded from this study. Detailed patient characteristics can be found in Table S1.

The patients were divided into groups according to tumor stage (II or III) and PRT status. The preoperative staging by MRI of patients with stage II tumors who had received PRT was checked in order to see if downstaging had been observed in any of these cases. Of these seven patients, one patient had stage III disease at the time of diagnosis (N1), one patient had not undergone a preoperative MRI (NX), and the remaining five patients were negative for regional lymph node metastases (N0). Therefore, in the majority of cases, the preoperative stage correlated with the pathological stage that was determined postoperatively. The clinical data was obtained from patient records, the survival data from the Population Register Center of Finland, and the cause of death for all the deceased from Statistics Finland. This study was a retrospective study that used plasma samples prospectively collected between 2000 and 2005. Participants provided written informed consent regarding plasma sample collection for future studies. This study 
Table 1. The proteins that passed the cutoff of a Mann-Whitney $U$ test $p$ value of less than 0.05 when samples from patients with stage II rectal cancer were analyzed according to preoperative radiotherapy (PRT) status (positive or negative)

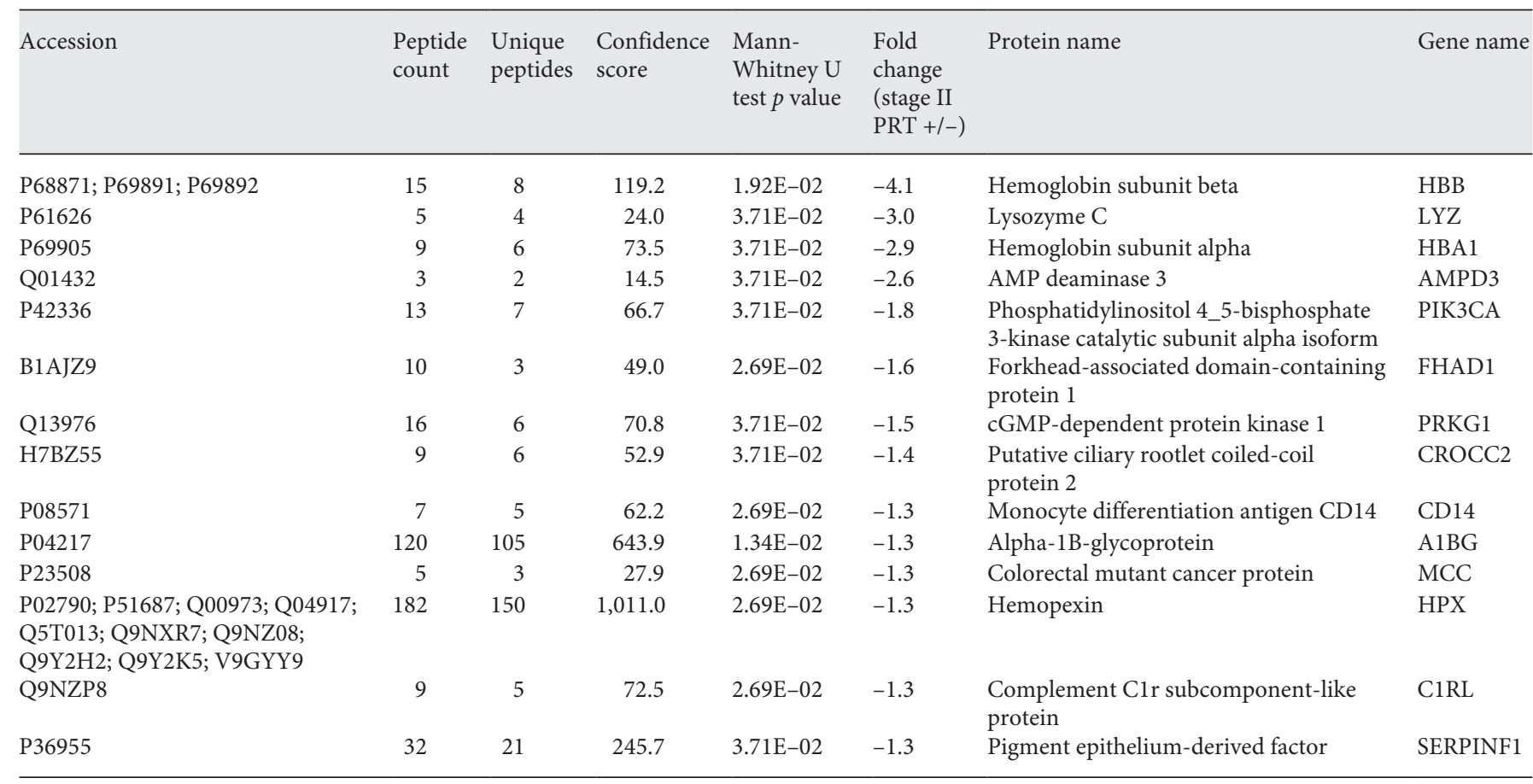

was planned as a small pilot study and the samples analyzed here may not be representative of a larger population. This study was approved by the Surgical Ethics Committee of Helsinki University Hospital (Dnro HUS 226/E6/06, extension TMK02 \$66 April 17, 2013) and was conducted according to the principles expressed in the Declaration of Helsinki.

Before the start of the study, we had assumed mean 1 to be 100 , mean 2 to be 200 , and the fold change to be 2 . The common standard deviation was assumed to be $75 \%$ of mean 1 . This gave us a sample size of 7 per group to differentiate the groups. However, the standard deviation common to both the groups was found to be larger after the study was carried out. Post-study, we have calculated the appropriate sample size for achieving $80 \%$ power for our top changing proteins to differentiate the two groups. For the stage II comparison, it was found to be 12 samples per group (median) and for the stage III comparison, it was 11 samples (median). When we calculated the power for our sample size, it was found to be in the range of $35-71 \%$ for stage II samples and $65-$ $84 \%$ for stage III samples, which is an acceptable power for a pilot study.

\section{Sample Processing}

The plasma samples were processed as previously described [18]. To summarize, the plasma samples were thawed and top 12 protein depletion was performed. The protein concentration in each sample was determined and the amount of plasma equivalent to $100 \mu \mathrm{g}$ of protein was aliquoted and dried. The dried plasma samples were then dissolved in Tris buffer containing urea and the samples were further processed and diluted. Trypsin digestion was performed overnight, after which $30 \mu \mathrm{g}$ of tryp- tic peptides were cleaned and the cleaned peptides were dissolved in formic acid containing $\mathrm{Hi} 3$ spike-in standard peptides for quantification.

\section{Ultra Performance Liquid Chromatography-Ultra Definition \\ Mass Spectrometry and Quantification}

UPLC-UDMS $^{\mathrm{E}}$ was performed as previously described [18]. Data were acquired with a Synapt G2-S HDMS (Waters Corporation, MA, USA) in data-independent acquisition fashion using UDMSE mode. Calibration was performed with sodium iodide clusters over a mass range of $50-2,500 \mathrm{~m} / \mathrm{z}$ by infusing $2 \mu \mathrm{g} / \mu \mathrm{L}$ sodium iodide solution in 50/50 2-propanol/water into the mass spectrometer.

\section{Data Analysis}

Data analysis and label-free quantification were performed as previously described $[18,19]$. The raw files were imported to Progenesis QI for proteomics (Nonlinear Dynamics, Newcastle, UK) and post-acquisition mass correction was performed when the raw data was imported into Progenesis with a lock mass ion of $\mathrm{M}+\mathrm{H}+$ $556.2771 \mathrm{~m} / \mathrm{z}$. Leucine enkephalin (C25H37O7, $1 \mathrm{ng} / \mu \mathrm{L}$ in 50:50 acetonitrile:water $+0.1 \%$ formic acid) was infused into the reference sprayer at $300 \mathrm{~nL} / \mathrm{min}$ for this correction.

Default parameters were used for peak picking and alignment and the peptide identification was done against Uniprot human FASTA sequences (release 2018_04). A ClpB protein sequence (CLPB_ECOLI [P63285]) was inserted for label-free quantification. Fixed modification at cysteine (carbamidomethyl) and variable at methionine (oxidation) were used. Trypsin was used as a digesting agent, and one missed cleavage was allowed. Fragment 


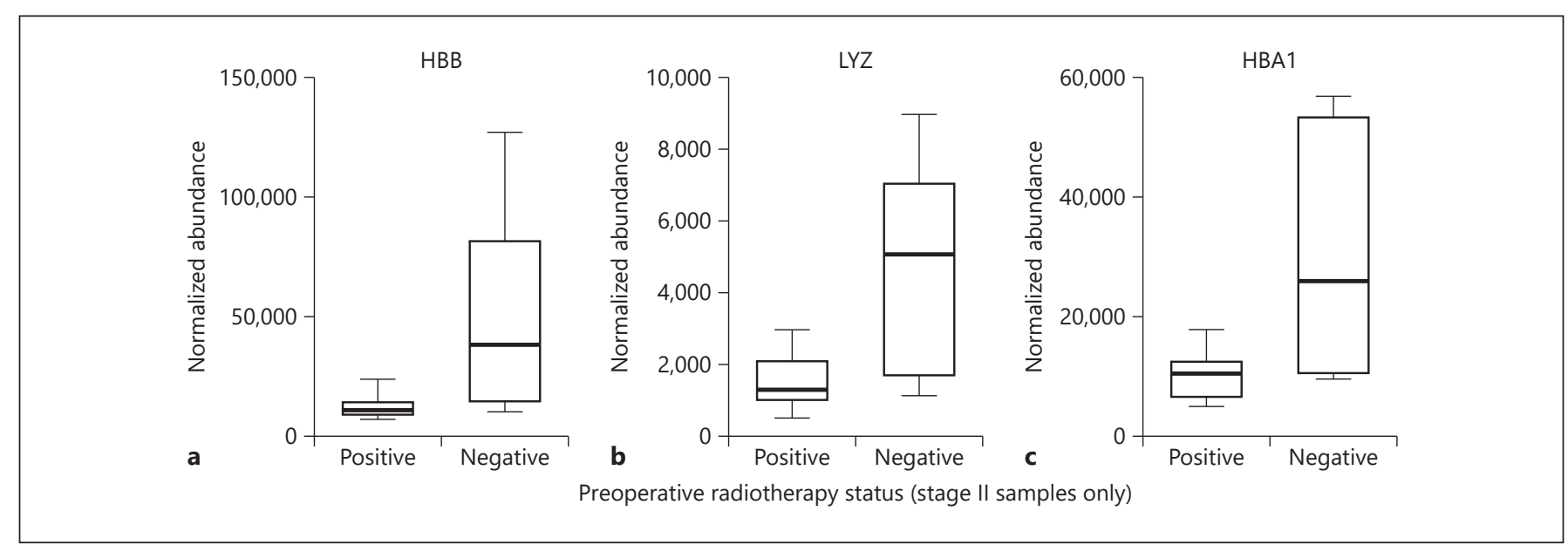

Fig. 1. The normalized abundance of the top three proteins according to fold change as box plots when samples from stage II rectal cancer patients were compared according to preoperative radiotherapy status. The box plots were plotted using the Tukey method and show the median, quartiles, and outliers. a Normalized abundance of HBB. b Normalized abundance of lysozyme C (LYZ). c Normalized abundance of hemoglobin subunit alpha (HBA1).
Fig. 2. The normalized abundance of the top two proteins according to fold change as box plots when samples from stage III rectal cancer patients were compared according to preoperative radiotherapy status. The box plots were plotted using the Tukey method and show the median, quartiles, and outliers. a Normalized abundance of serum amyloid A1 (SAA1). b Normalized abundance of synapsin 2 (SYN2).

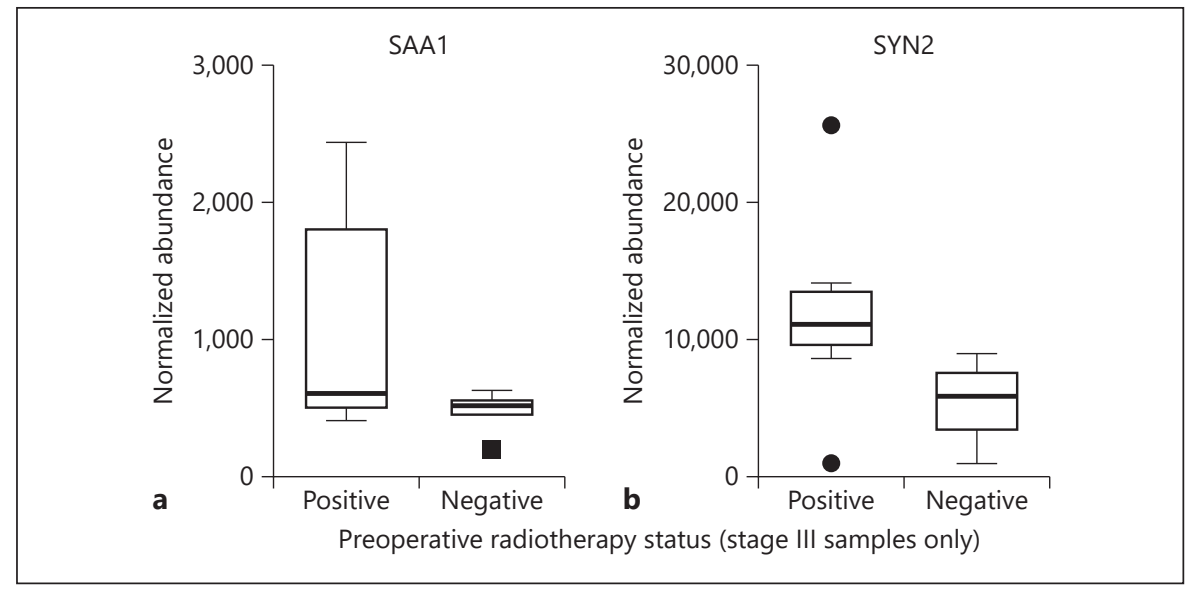

and peptide error tolerances were set to automatic settings and the false discovery rate was set to less than $2 \%$. For ion matching, one or more ion fragments per peptide, three or more fragments per protein, and one or more peptides per protein were required (the default parameters). The mass spectrometry proteomics data have been deposited to the ProteomeXchange Consortium via the PRIDE [20] partner repository with the dataset identifier PXD013150 and 10.6019/PXD013150.

\section{Further Analysis}

The differences between the groups were analyzed using the Mann-Whitney $U$ test or T test. The Mann-Whitney $U$ test was used when the abundances of the majority $(>50 \%)$ of proteins were not normally distributed, while the T test was used when the abundances of the majority $(>50 \%)$ of proteins were normally distributed. Box plots were drawn using GraphPad Prism 7 (GraphPad Software, La Jolla, CA, USA).

\section{Results}

In this study, we analyzed the plasma samples from 30 rectal cancer patients into groups based on whether the patients had received PRT or not. This was done in order to study if PRT leads to differences in plasma protein expression between patients. We compared patients according to PRT status separately depending on whether patients had stage II or III rectal cancer.

Among stage II rectal cancer patients, 7 received PRT and 6 patients did not. A total of 14 proteins passed the cutoff of a Mann-Whitney $U$ test $p$ value of less than 0.05 (Table 1) when samples from these patients were compared according to PRT status. All 14 proteins displayed 
Table 2. The proteins that passed the cutoff of a T test $p$ value of less than 0.05 when samples from patients with stage III rectal cancer were analyzed according to PRT status (positive or negative)

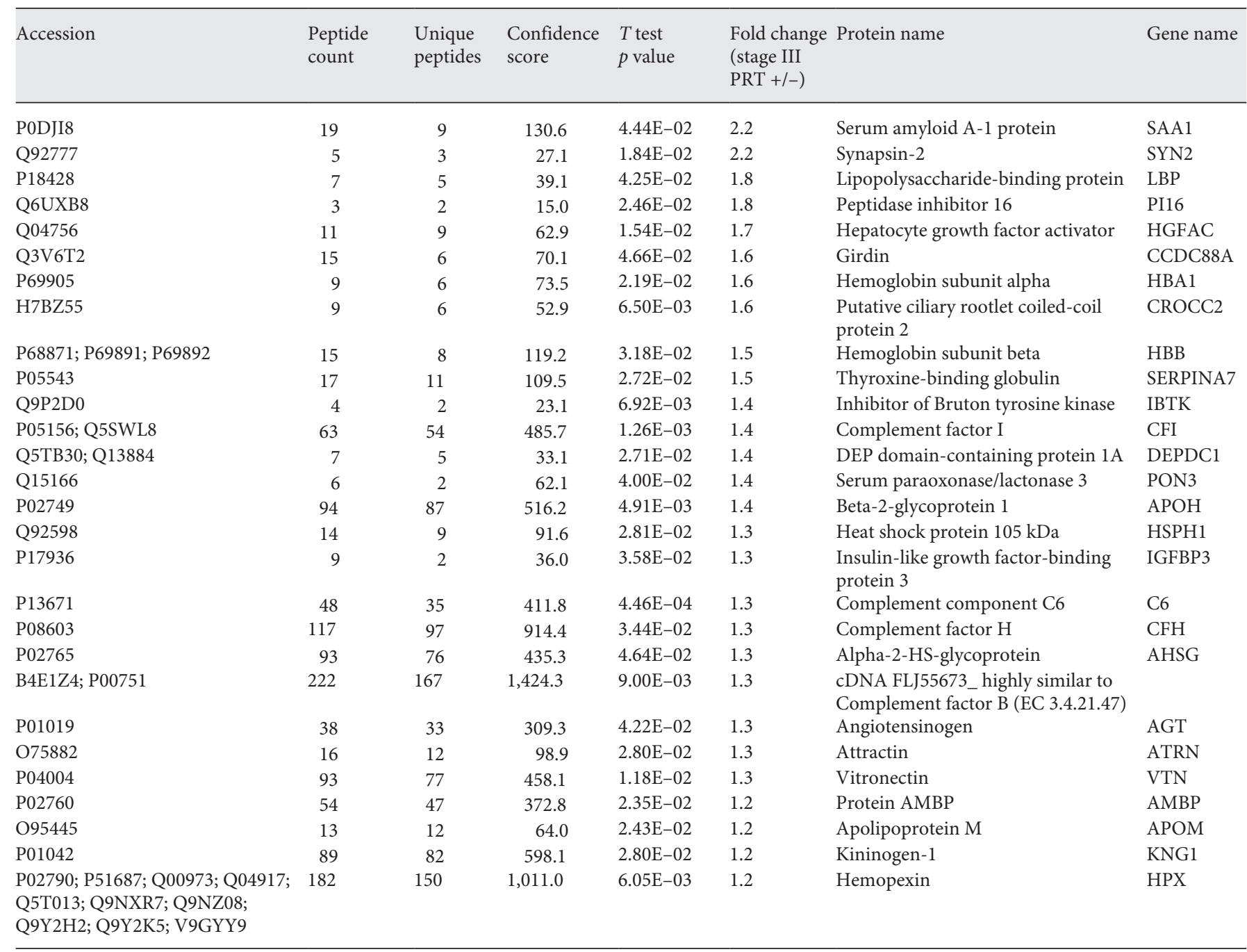

higher levels in the plasma of stage II rectal cancer patients who were PRT negative. The protein with the largest fold change (4.1) was hemoglobin subunit beta (HBB), which displayed higher plasma levels in patients who were negative for PRT. Figure 1 shows the normalized abundance of the top three proteins in stage II rectal cancer patients who were PRT positive or negative as box plots.

Among stage III rectal cancer patients, 9 patients received PRT and 8 did not. In this group, a total of 28 proteins passed the cutoff of a T test $p$ value of less than 0.05 (Table 2) when samples from these patients were compared. In contrast to the proteins identified in stage II rectal cancer patients, all of these 28 proteins displayed higher levels in the plasma of stage III rectal cancer patients who received PRT. The protein with the largest fold change (2.2) was serum amyloid A-1 (SAA1), which had higher plasma levels in patients who were positive for PRT. Figure 2 shows the normalized abundance of the top two proteins in stage III rectal cancer patients who were PRT positive or negative as box plots.

\section{Discussion}

In this study, we have analyzed the differences in plasma protein expression between a small group of rectal cancer patients who were divided into groups based on PRT status 
and tumor stage (II or III). This was done in order to study the differences in plasma protein expression in a stage-specific manner. Here, we discovered 14 plasma proteins whose levels differed significantly depending on PRT status in stage II rectal cancer patients, and 28 plasma proteins whose levels differed significantly between stage III rectal cancer patients. Levels of three proteins, $\mathrm{HBB}$, hemoglobin subunit alpha (HBA1), and hemopexin (HPX), were significantly altered between samples from both stage II and III rectal cancer patients. However, in stage II patients, levels of these proteins were higher in patients who were PRT negative, while in stage III patients, they were higher in patients who were PRT positive. These findings indicate that PRT can have different effects on plasma protein expression depending on tumor stage.

We have previously compared plasma samples from CRC patients according to patient survival, where patients were divided into groups based on whether they survived or died due to CRC. Patients who died due to stage II CRC were found to have higher plasma levels of HBB than patients who survived having stage II CRC (unpublished data; the raw mass spectrometric data can be found in the PRIDE [20] repository with the dataset identifier PXD013150). These findings indicate that higher plasma levels of HBB may be linked to poor outcome in patients with stage II CRC. PRT has also been found to improve survival [6], and we have found that stage II rectal cancer patients who did not receive PRT had higher levels of plasma HBB (fold change of 4.1), which further supports the conclusion that higher levels of HBB may affect patient survival. A study by Zheng et al. [21] showed that expression of HBB by circulating tumor cells promotes cell survival during hematogenous dissemination in breast, prostate, and lung cancer. Circulating tumor cells displayed an elevated expression of $\mathrm{HBB}$, which also promoted the initiation of distant metastases in mouse models of breast cancer. The authors also found that HBB protects epithelial cancer cells from reactive oxygen species (ROS)-induced apoptosis [21]. Additional studies are needed in order to investigate the role of $\mathrm{HBB}$ in CRC and if elevated levels of HBB could directly affect factors such as cancer aggressiveness and metastasis. HBB could for example be studied in a larger series of patients using commercially available ELISA kits in order to examine a possible link between $\mathrm{HBB}$ expression and factors such as response to radiotherapy or patient survival.

Radiotherapy causes DNA damage either through the ionization of DNA or the generation of ROS, which leads to single- and double-strand breaks, as well as other DNA lesions $[22,23]$. The main cell death mechanisms follow- ing exposure to irradiation are radiation-induced apoptosis and mitotic catastrophe (cell death that occurs during or due to aberrant mitosis) [24]. Expression of hemoglobin subunit epsilon (HBE1, also known as epsilon globin), which encodes a subunit of hemoglobin, is normally silenced in adults. However, HBE1 has been found to be overexpressed in CRC cell lines made resistant to radiotherapy by exposure to ionizing radiation over several weeks. These cells displayed decreased ROS production and cell death when subsequently exposed to radiation [25]. Hemoglobin, which is a complex of heme and globin, has been discovered to increase proliferation in CRC cells through the production of ROS [26]. In this study, the plasma levels of both HBA1 and HBB, as well as the levels of HPX, were altered between rectal cancer patients depending on PRT status. HPX is a plasma protein that binds heme released from heme-binding proteins such as hemoglobin [27]. These results indicate that the generation of ROS caused by radiotherapy directly affects heme levels and may also affect different aspects of CRC, such as cell proliferation and survival.

There is a lack of proteomic studies investigating how PRT affects the plasma proteome and profiling the differences between rectal cancer patients who did or did not receive PRT. In this study, we have shown that the levels of multiple plasma proteins differ between rectal cancer patients depending on PRT status, with stage-specific differences being seen. Fold changes were generally larger for the proteins whose levels differed between stage II rectal cancer patients, ranging from 1.3 to 4.1 (compared to from 1.2 to 2.2 for stage III patients), while the levels of more proteins differed significantly between stage III than stage II rectal cancer patients (28 vs. 14 ). Our results show that PRT, although localized to the pelvis for rectal cancer patients, leads to significant changes in plasma protein expression that can be detected and quantified from easily obtainable blood samples. Seeing as PRT significantly affects plasma protein expression, PRT status has to be taken into consideration when studying the plasma proteome. It is important to be aware of the fact that PRT leads to changes in the levels of specific plasma proteins, especially if these proteins are the subjects of future studies in situations where patients may have received PRT.

Although this study analyzed the plasma samples of a relatively small cohort of 30 rectal cancer patients, we have identified multiple plasma proteins whose levels differ according to PRT status in patients with stage II or III rectal cancer. Whereas previous studies have to a large extent focused on identifying proteins that can function as biomarkers of response to radiotherapy and proteins 
involved in resistance to radiotherapy, this study has investigated the overall differences in plasma protein expression depending on whether patients did or did not receive PRT. This study shows that PRT leads to widespread, significant changes in plasma protein expression. This is something that accordingly must be taken into account in future studies involving plasma proteins where patients may have received PRT. These results suggest that PRT may also significantly affect the plasma protein profiles of patients with other types of cancer treated with PRT, such as breast, prostate, and cervical cancer.

Further studies are needed to investigate if PRT status also needs to be taken into account in relevant studies of these types of cancer. The findings of this study support the further analysis of proteins discovered here, such as $\mathrm{HBB}$, in a larger series of samples using ELISA assays to study the link between protein expression and factors such as PRT response and patient survival. Additionally, future studies should aim to analyze the plasma protein expression in a larger set of samples collected before and after PRT from the same patients, which was unfortunately not possible here, as no plasma samples were collected prior to PRT. However, this pilot study has shown that PRT significantly affects plasma protein expression and is something that should be further investigated.

\section{Acknowledgements}

The authors would like to thank the funders mentioned.

\section{Statement of Ethics}

Participants provided written informed consent regarding plasma sample collection for future studies. This study was approved by the Surgical Ethics Committee of Helsinki University Hospital (Dnro HUS 226/E6/06, extension TMK02 \$66 April 17, 2013) and was conducted according to the principles expressed in the Declaration of Helsinki.

\section{Disclosure Statement}

The authors have no conflicts of interest to declare.

\section{Funding Sources}

This study was supported by grants from the University of Helsinki, Helsinki University Hospital and HUSLAB, Helsinki University Hospital, Helsinki University Central Hospital Research Funds, The Sigrid Jusélius Foundation, The Finnish Cancer Foundation, and Finska Läkaresällskapet. The funders had no role in study design, data collection and analysis, decision to publish, or preparation of the manuscript.

\section{Author Contributions}

Study conception and design: M.H., S.J., M.S., A.R., R.R., and C.H. Acquisition of data: M.H., S.J., and T.T. Analysis and interpretation of data: M.H., S.J., and M.S. Drafting of manuscript: M.H. Revision: M.H., S.J., M.S., T.T., A.R., R.R., C.H. Resources: A.R., R.R., C.H.

\section{References}

1 Bray F, Ferlay J, Soerjomataram I, Siegel RL, Torre LA, Jemal A. Global cancer statistics 2018: GLOBOCAN estimates of incidence and mortality worldwide for 36 cancers in 185 countries. CA Cancer J Clin. 2018;68(6):394424.

2 Tamas K, Walenkamp AM, de Vries EG, van Vugt MA, Beets-Tan RG, van Etten B, et al. Rectal and colon cancer: not just a different anatomic site. Cancer Treat Rev. 2015;41(8):671-9.

3 Hong TS, Clark JW, Haigis KM. Cancers of the colon and rectum: identical or fraternal twins? Cancer Discov. 2012;2(2):117-21.

4 Kapiteijn E, Marijnen CA, Nagtegaal ID, Putter H, Steup WH, Wiggers T, et al. Preoperative radiotherapy combined with total mesorectal excision for resectable rectal cancer. $\mathrm{N}$ Engl J Med. 2001;345(9):638-46.

5 Folkesson J, Birgisson H, Pahlman L, Cedermark B, Glimelius B, Gunnarsson U. Swedish Rectal Cancer Trial: long lasting benefits from radiotherapy on survival and local recurrence rate. J Clin Oncol. 2005;23(24):5644-50.
6 Camma C, Giunta M, Fiorica F, Pagliaro L, Craxi A, Cottone M. Preoperative radiotherapy for resectable rectal cancer: a meta-analysis. JAMA. 2000;284(8):1008-15.

7 Peng J, Gygi SP. Proteomics: the move to mixtures. J Mass Spectrom. 2001;36(10):1083-91.

8 Hanash SM, Pitteri SJ, Faca VM. Mining the plasma proteome for cancer biomarkers. $\mathrm{Na}$ ture. 2008;452(7187):571-9.

9 Anderson NL, Anderson NG. The human plasma proteome. Mol Cell Proteomics. 2002; 1(11):845-67.

10 Chang L, Graham P, Hao J, Bucci J, Malouf D, Gillatt D, et al. Proteomics discovery of radioresistant cancer biomarkers for radiotherapy. Cancer Lett. 2015;369(2):289-97.

11 Lacombe J, Azria D, Mange A, Solassol J. Proteomic approaches to identify biomarkers predictive of radiotherapy outcomes. Expert Rev Proteomics. 2013;10(1):33-42.
12 Bowden DL, Sutton PA, Wall MA, Jithesh PV, Jenkins RE, Palmer DH, et al. Proteomic profiling of rectal cancer reveals acid ceramidase is implicated in radiation response. J Proteomics. 2018;179:53-60.

13 Repetto O, De Re V, De Paoli A, Belluco C, Alessandrini L, Canzonieri V, et al. Identification of protein clusters predictive of tumor response in rectal cancer patients receiving neoadjuvant chemo-radiotherapy. Oncotarget. 2017;8(17):28328-41.

14 Chauvin A, Wang CS, Geha S, Garde-Granger P, Mathieu AA, Lacasse V, et al. The response to neoadjuvant chemoradiotherapy with 5-fluorouracil in locally advanced rectal cancer patients: a predictive proteomic signature. Clin Proteomics. 2018;15:16.

15 Smith FM, Gallagher WM, Fox E, Stephens RB, Rexhepaj E, Petricoin EF, 3rd, et al. Combination of SELDI-TOF-MS and data mining provides early-stage response prediction for rectal tumors undergoing multimodal neoadjuvant therapy. Ann Surg. 2007;245(2):259-66. 
16 Allal AS, Kahne T, Reverdin AK, Lippert H, Schlegel W, Reymond MA. Radioresistancerelated proteins in rectal cancer. Proteomics. 2004;4(8):2261-9.

17 Arnold M, Sierra MS, Laversanne M, Soerjomataram I, Jemal A, Bray F. Global patterns and trends in colorectal cancer incidence and mortality. Gut. 2017;66(4):683-91.

18 Holm M, Saraswat M, Joenvaara S, Ristimaki A, Haglund C, Renkonen R. Colorectal cancer patients with different C-reactive protein levels and 5-year survival times can be differentiated with quantitative serum proteomics. PLoS One. 2018;13(4):e0195354.

19 Silva JC, Gorenstein MV, Li GZ, Vissers JP, Geromanos SJ. Absolute quantification of proteins by LCMSE: a virtue of parallel MS acquisition. Mol Cell Proteomics. 2006;5(1): $144-56$.
20 Deutsch EW, Csordas A, Sun Z, Jarnuczak A, Perez-Riverol Y, Ternent T, et al. The ProteomeXchange consortium in 2017: supporting the cultural change in proteomics public data deposition. Nucleic Acids Res. 2017;45(D1): D1100-D6.

21 Zheng Y, Miyamoto DT, Wittner BS, Sullivan JP, Aceto N, Jordan NV, et al. Expression of beta-globin by cancer cells promotes cell survival during blood-borne dissemination. Nat Commun. 2017;8:14344.

22 Baskar R, Dai J, Wenlong N, Yeo R, Yeoh KW. Biological response of cancer cells to radiation treatment. Front Mol Biosci. 2014;1: 24.
23 Lomax ME, Folkes LK, O’Neill P. Biological consequences of radiation-induced DNA damage: relevance to radiotherapy. Clin Oncol (R Coll Radiol). 2013;25(10):578-85.

24 Eriksson D, Stigbrand T. Radiation-induced cell death mechanisms. Tumour Biol. 2010; 31(4):363-72.

25 Park SY, Lee SJ, Cho HJ, Kim JT, Yoon HR, Lee KH, et al. Epsilon-globin HBE1 enhances radiotherapy resistance by down-regulating BCL11A in colorectal cancer cells. Cancers (Basel). 2019;11(4).

26 Lee RA, Kim HA, Kang BY, Kim KH. Hemoglobin induces colon cancer cell proliferation by release of reactive oxygen species. World J Gastroenterol. 2006;12(35):5644-50.

27 Hvidberg V, Maniecki MB, Jacobsen C, Hojrup P, Moller HJ, Moestrup SK. Identification of the receptor scavenging hemopexin-heme complexes. Blood. 2005;106(7):2572-9. 